\title{
Immunohistochemical Expression of Heat Shock Proteins in the Mouse Periodontal Tissues due to Orthodontic MECHANICAL STRESS*
}

\author{
R. Muraoka ${ }^{1,2}$, K. Nakano ${ }^{1,3}$, S. Kurihara ${ }^{3}$, K. Yamada ${ }^{2,4}$, T. Kawakami1,3 \\ ${ }^{1}$ Hard Tissue Pathology Unit, Matsumoto Dental University Graduate School of Oral Medicine, Shiojiri, Japan \\ ${ }^{2}$ Department of Orthodontics, Matsumoto Dental University School of Dentistry, Shiojiri, Japan \\ ${ }^{3}$ Matsumoto Dental University Institute for Oral Science, Shiojiri, Japan \\ ${ }^{4}$ Clinical Evaluation Unit, Matsumoto Dental University Graduate School of Oral Medicine, Shiojiri, Japan
}

\begin{abstract}
The histopathology of periodontal ligament of the mouse subjected to mechanical stress was studied. Immunohistochemical expressions of HSP27 and pHSP27 were examined. Experimental animals using the maxillary molars of ddY mouse by Waldo method were used in the study. A separator was inserted to induce mechanical stress. After 10 minutes, 20 minutes, 1 hour, 3 hours, 9 hours and 24 hours, the regional tissues were extracted, fixed in $4 \%$ paraformaldehyde and $0.05 \mathrm{M}$ phosphate-buffered fixative solution. Paraffin sections were made for immunohistochemistry using HSP27 and p-HSP27. In the control group, the periodontal ligament fibroblasts expressed low HSP27 and p-HSP27. However, in the experimental group, periodontal ligament fibroblasts expressed HSP27 10 minutes after mechanical load application in the tension side. The strongest expression was detected 9 hours after inducing mechanical load. p-HSP27 was also expressed in a time-dependent manner though weaker than HSP27. The findings suggest that HSP27 and p-HSP27 were expressed for the maintenance of homeostasis of periodontal ligament by the activation of periodontal ligament fibroblasts on the tension side. It also suggests that these proteins act as molecular chaperones for osteoblast activation and maintenance of homeostasis.
\end{abstract}

Key words: Heat shock protein, HSP27, periodontal ligament cells, mechanical stress, orthodontic tooth movement

\section{INTRODUCTION}

Orthodontic treatment results to mechanical stress inducing reorganization of periodontal ligament (PDL) collagen bundles. As a result, the periodontal tissues supporting the movement of the tooth called pressure side react with the appearance of osteoclasts responsi-

* This study was conducted as thesis fulfillment for the PhD degree in Dentistry of Dr Rina Muraoka, under the supervision of Professor Toshiyuki Kawakami, Hard Tissue Pathology Unit, Department of Hard Tissue Research, Matsumoto Dental University Graduate School of Oral Medicine. ble for bone resorption. On opposite side, generally known as tension side, activation of osteoblasts adds attachment to bone at the same time reorganization occurs in periodontal tissues. These actions would result to tooth movement. Mechanical stress transferred to the PDL causes tissue reaction causing tooth movement. This reaction of PDL is for the maintenance of homeostasis. In recent years, histological response and cellular differentiation, as well production of transcription factors regulating various morphogenetic phenomenon became more widely studied [1,2]. It has become clear that maintaining homeostasis can lead to periodontal tissue remodeling and expression of active molecules in response to various mechanical stress and inflammation [3-8].

Traditionally, major proteins expressed in various tissues and organs due to mechanical load and stimulation of cytotoxic stress that is transiently expressed are known as heat shock proteins (HSPs) [9]. HSPs are not only induced by heat shock but also by various pathological changes such as ischemia, infection and inflammation; radiation, physical stress such as light, enzyme; heavy metal ions like arsenic, ethanol, free radicals, amino acid derivatives, various chemicals induced by stress $[10,11]$. HSPs are thought to be involved in the regulation of cell function and defense as well as against injury to the cell [12-14]. However, its dynamic role in PDL and tooth movement as well as in the regulatory cell response against stimuli from experimental injury has not been investigated. Shigehara, et al. [15] studied the expressions of various mRNAs in the dental pulp after subjecting to mechanical stress during orthodontic movement but the expression of HSP was not mentioned. In our knowledge and in search of literatures, HPS expression in periodontal tissues is very limited. Maeda et al. [10] reported that cultured human PDL-derived fibroblast-like cells when subjected to continuous compression expressed HSP60, 70 and 90. Also, Okazaki et al. [11] reported the increased expression of HSP47, 60 and 70 by in vitro cyclic loading of stretching force in human PDL fibroblasts. Furthermore, Araujo et al. [16] mentioned that in culture experiment, microarray revealed that only HSP70 increased when human PDL fibroblasts were exposed to mechanical stress. HSP is classified into two types de- 
pending on the molecular weight. The comparison between HSP70 and other low- molecular weight HSPs have not been clearly elucidated [17].

In this study, the immunohistochemical expression of HSP specifically HSP27 and phospphorylated HSP27 (p-HSP27) were simultaneously examined after subjecting the periodontal tissues to mechanical stress by orthodontic movement. Histopathological changes of PDL in pressure and tension sides were studied using mouse experimental animals.

\section{Materials And Method}

\section{1) EXPERIMENTAL ANIMALS}

A total of 30,8 week-old ddY male mice weighing 35 $\pm 5 \mathrm{~g}(30-40 \mathrm{~g})$ were purchased from Japan SLC Co. Ltd. (Hamamatsu, Japan). The mice were housed in metal containers lined with a floor mat (Paper clean: Peparlet Co., Ltd., Shizuoka, Japan) in air-conditioned room $\left(24 \pm 1^{\circ} \mathrm{C}\right)$. The animals were fed freely with water and solid diet during breeding (Picolab Rodent Diet 20: Japan SLC Inc., Hamamatsu, Japan).

\section{2) EXPERIMENTAL METHODS}

The experimental method was based on our previous reports [3, 4]. The Waldo method of inducing mechanical stress-load in mouse periodontal tissues was followed [18]. Prior to mechanical load induction, inhalation anesthesia was used using isoflurane (Isoflu: Dainippon Sumitomo Pharma Co., Osaka, Japan) and gas-air mixture (4.0\% concentration of pre-anesthesia). In order to achieve a lasting and stable anesthesia, adjustable constant flow rate gas anesthesia system (Farmer Biometrical Ltd., Laboratory, Osaka, Japan) for small laboratory animals was used. Under general anesthesia, the mouse was locked in a sitting position where the upper body was held on the experiment bench top. Isoflurane inhalation anesthesia was set to the nose for the maintenance of general anesthesia $(1.0 \%$ concentration) during the experiment. To keep the mouth open, the maxilla was fixed with a thread tied on the upper incisor above the bench and rubber was used to fix the mandible. While the mouth was open, the separator was inserted to the maxillary molar to induce persistent mechanical stress by Waldo method (Fig. 1, 2). The separator was used with $2 \times 2 \mathrm{~mm}$ heavy force rubber dam sheet (Ivory, Premium rubber Dam Pure Latex: Heraeus Kulzer GmbH \& Co. KG, Hanau, Germany). The maxilla has 3 molars; first molar (M1), second molar (M2) and third molar (M3) from mesial side. In addition, M1 and M2 have 3 roots and M3 has 2 to 3 roots. Separator was inserted between M1 and M2 of right maxillary molars to ensure the mechanical stress due to pressure over a period of time. Experimental animals were divided into 6 groups; 10 minutes, 20 minutes, 1 hour, 3 hours, 9 hours, 24 hours having 5 animals per group. Table 1 shows number of the speci- mens in each subgroup. After each experimental time, the anesthetized animals were killed by ether inhalation, and the relevant tissues were removed as one block. The periodontal tissues of the left maxillary molar region (untreated side) were used as controls. In this experiment, the distal buccal root of the maxillary first molar was the observation part. The current experiment was performed according to the Guidelines for Animal Experiments of Matsumoto Dental University.
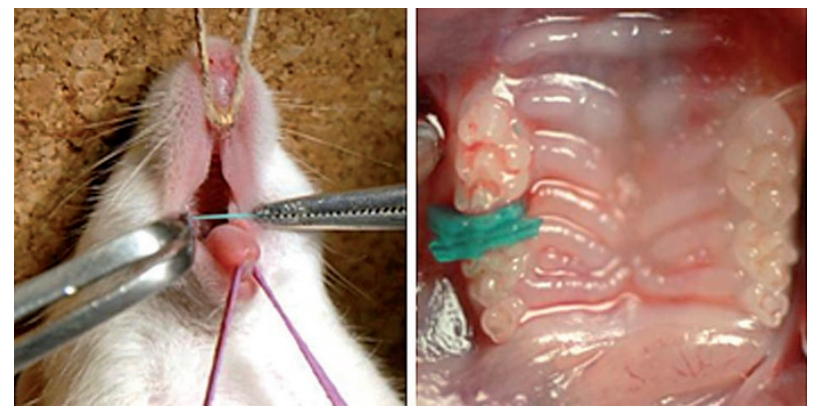

Fig. 1. Mouse placed on the experimental bench (Left) and the situation of the insertion (right).

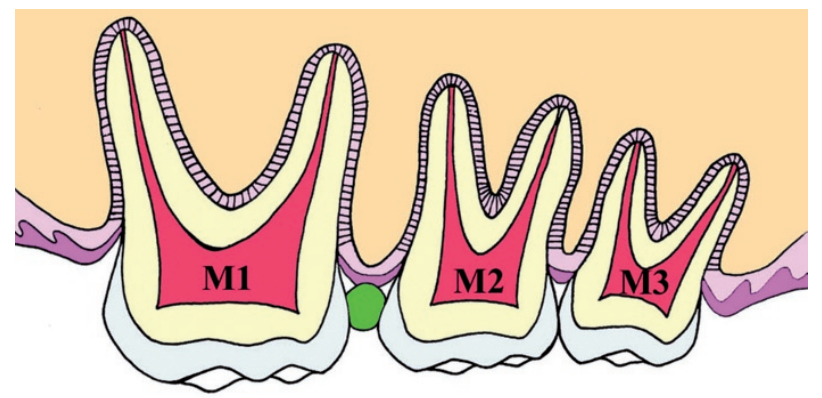

Fig. 2. Diagram of the insertion part of the separator (green oval image)

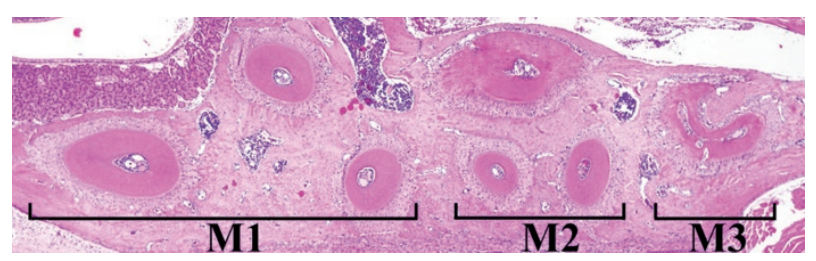

Fig. 3. Horizontal histopathological image of experimental specimen as shown in Fig. 2.

\section{TISSUE PREPARATION}

The maxillary molar with the periodontal tissues were removed promptly and fixed in $4 \%$ paraformaldehyde with $0.05 \mathrm{M}$ phosphate buffer for 24 hours and decalcified in 10\% EDTA solution for 3 weeks. After embedding in paraffin, serial sections of $5 \mu \mathrm{m}$ were made for histopathological and immunohistochemical studies. Figure 3 shows the histopathological sections of M1, M2 and M3.

Table 1. Experimental Periods and Number of Specimens

\begin{tabular}{lcccccccc}
\hline Periods & Cont & $10 \mathrm{~min}$ & $20 \mathrm{~min}$ & $1 \mathrm{hr}$ & $3 \mathrm{hrs}$ & $9 \mathrm{hrs}$ & $24 \mathrm{hrs}$ & Total \\
Numbers & 5 & 5 & 5 & 5 & 5 & 5 & 5 & 35
\end{tabular}




\section{IMMUNOHISTOCHEMISTRY}

Slides underwent deparaffinization and then subjected to pre-treatment for 30 minutes in incubator at $60^{\circ} \mathrm{C}$. Immunohistochemistry was done with Dako Envision + Kit-K4006 (Dako, Glostrup, Denmark). Primary antibodies used were HSP27 mouse monoclonal antibody (HSP27 (SPM252): sc-65567, Santa Cruz Biotechnology, Inc., Santa Cruz, CA, USA, with a concentration of 1:5000) and mouse p-HSP27 rabbit polyclonal antibody (p-HSP27 (Ser 15): sc-101699, Santa Cruz Biotechnology, Inc., Santa Cruz, CA, USA, with a concentration of $1: 5000)$. Specimens were counterstained with hematoxylin. For negative controls, specimens were stained without primary antibodies.

\section{RESULTS}

\section{HistopatholOGICAL EXAMINATION}

\section{Control group}

When distal buccal root of the upper first molar was horizontally examined, the alveolar bone socket was in oval shape with a long diameter of $550 \mu \mathrm{m}$ and a short diameter of $450 \mu \mathrm{m}$ though a small variation of size between the samples existed. The tooth root was located almost in the center of the alveolar socket. The mesial side has a slight deviation where the PDL space is about $50 \mu \mathrm{m}$. The distal PDL in between the alveolar bone and the distal buccal root of the left maxillary first molar were relatively arranged in order (Fig. 4, CH, C-T). Spindle-shaped fibroblasts with oval nuclei can be seen along the PDL fibers. However, some sites also showed PDL fibroblasts positioned in a slightly different direction. Interspersed with the collagen bundles are capillaries filled with few erythrocytes. The root surface of acellular cementum is darkly stained with hematoxylin. The alveolar bone contains few flattened osteoblasts with oval nucleus.

In contrast, in the mesial PDL of the distal buccal root, the PDL fibroblasts were observed in a diagonal orientation (Fig. 4, C-P). Spindle-shaped PDL fibroblasts with oval nuclei were relatively even. The cementum and alveolar bone surfaces were the same as the distal segment.

\section{Experimental group}

PDL in the tension side

Changes in the distal buccal root of maxillary first molar were markedly different in the experimental group. The side near the separator (distal PDL of the distal buccal root of right maxillary first molar) is the tension side; the opposite side (mesial PDL of the distal buccal root of the maxillary first molar) is the pressure side.

In the 10 minutes group, the tooth moved slightly to the mesial. However, changes in the width of the PDL were not clear. The nucleus of PDL fibroblasts in the tension side was elongated. Their cytoplasm and nucleus were both slightly elongated.

In the 20 minutes group, difference between the tension and pressure side became apparent (Fig. 4, $20 \mathrm{~m}-\mathrm{H})$. The PDL in the tension was arranged in a radial direction. The PDL fibroblasts have been strongly stretched, spindle in shape and the nucleus is flattened or elliptical in shape. Also, congested capillaries are seen in the PDL (Fig. 4, 20m-T).

In the 1 hour group, the root moved further to the mesial direction (Fig. 4, 1h-H). The PDL width created a greater traction and the fibers had been stretched considerably. The nucleus was intensely stained with hematoxylin and became pyknotic. Destroyed collagen bundles in between gaps were observed. Nucleus disappeared in destroyed collagen bundles. The cells that have been stretched have wavy appearance. Some PDL fibroblasts with round nucleus were also observed. Congested capillaries in between collagen bundles increased. Osteoblasts lining the surface of alveolar bone are oval in shape (Fig. 4, 1h-T).

In the 3 hours group, the root continued to move mesially and the width of the PDL space increased (Fig. 4, 3h-H). The expected extension of PDL caused gaps in between fibers. The cytoplasm of spindleshaped fibroblasts showed oval nuclei. The number of fibroblasts decreased compared to the 1 hour group. In particular, reduced chromatin condensation was observed in flattened cells. Congested capillaries are seen. Oval-shaped osteoblasts are observed lining the surface of the alveolar bone (Fig. 4, 3h-T).

In the 9 hours group, the root stopped moving in the mesial direction and the width of the PDL did not further increase and was maintained similar to the width observed in the 3 hours group. The gap in between collagen bundles was reduced. PDL fibroblasts are short, spindle in shape with oval nucleus. On the other hand, congested capillaries can still be observed.

In the 24 hours group, PDL cells are spindle in shape with thick oval nucleus. Osteoblasts lining the alveolar bone surface are oval or short cuboidal in shape. Dilated vessels and scattered hemorrhages can be observed (Fig. 4, 24h-H, 24h-T).

PDL in the pressure side

In the 10 minutes group, there were significant differences in the width in the tension and pressure sides of the PDL but showed little changes in cell morphology and arrangement of PDL fibroblasts. The nucleus was compressed and the intercellular space was reduced due to increased cell density.

In the 20 minutes group, the disorder in cell morphology of PDL fibroblasts became clearer in the pressure side. Nuclear form of PDL fibroblasts became further irregular, nuclear core became partly pyknotic. The osteoblasts have scanty cytoplasm, oval nuclei; some were flattened but the number of cells was lower compared to the 10 minutes group (Fig. 4, 20m-P).

In the 1 hour group, the PDL space is narrower; PDL fibroblasts are arranged in layers around the root. Moreover, the PDL cells showed various degenerative changes. The cytoplasm of PDL fibroblasts became eosinophilic, nucleus is flat, karyopyknosis was evident. Furthermore, osteoblasts lining the bone surface were reduced in number; the cytoplasm and nucleus became flattened by pressure and distinction among surrounding fibroblasts became difficult (Fig. 4, 1h-P).

In the 3 hours group, the PDL space further became narrower, degenerative changes were more severe that distinction of PDL cells was difficult. PDL 

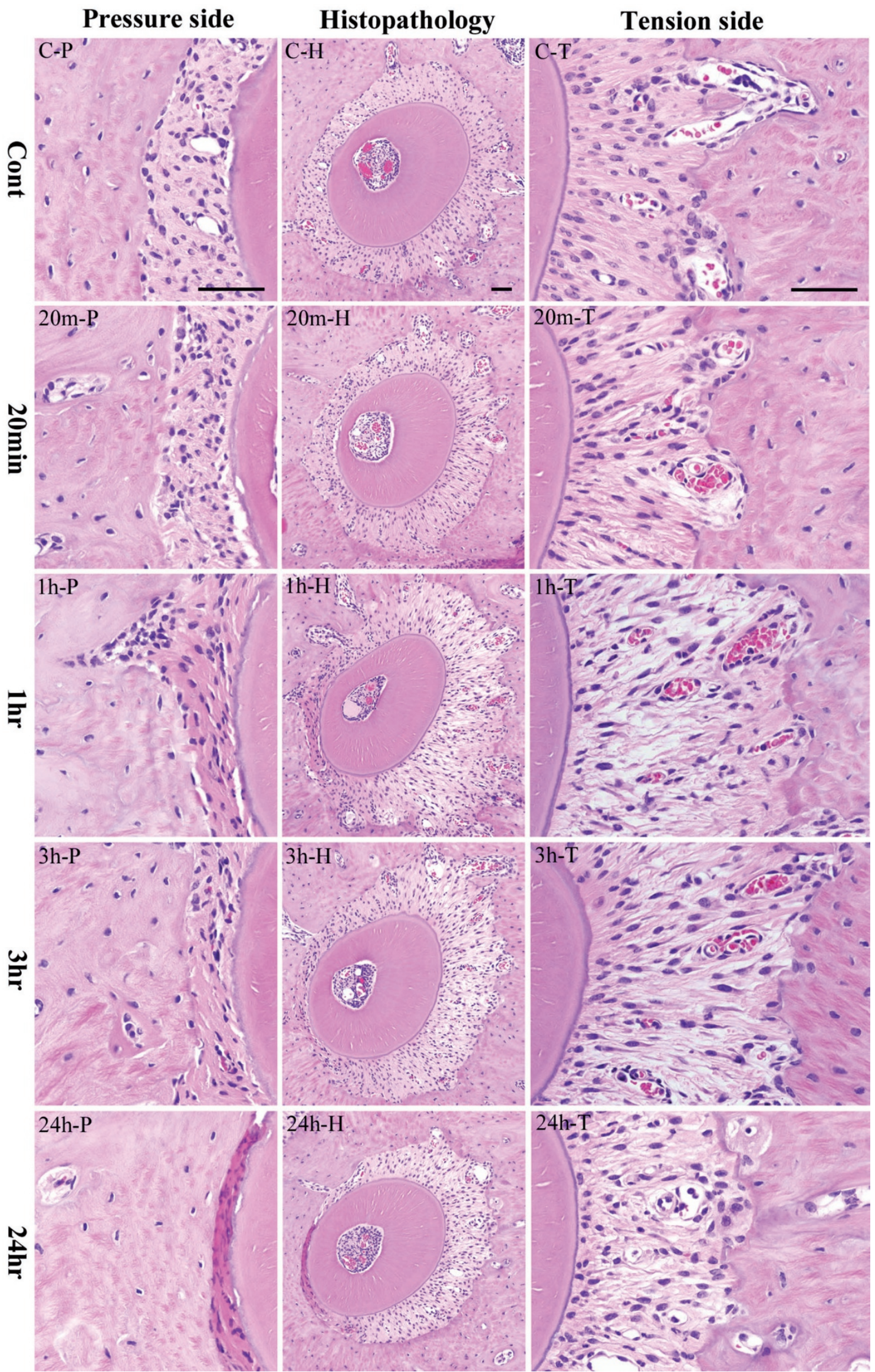

Fig. 4. Histopathological results. Center column shows histopathological image of the alveolar bone socket morphology. Left column is high power-view of pressure side and right column tension side. The most upper row is the control group and the following is the experimental groups of 20 minutes, 1 hour, 3 hours and 24 hours. The inset bar indicates $50 \mu \mathrm{m}$. 

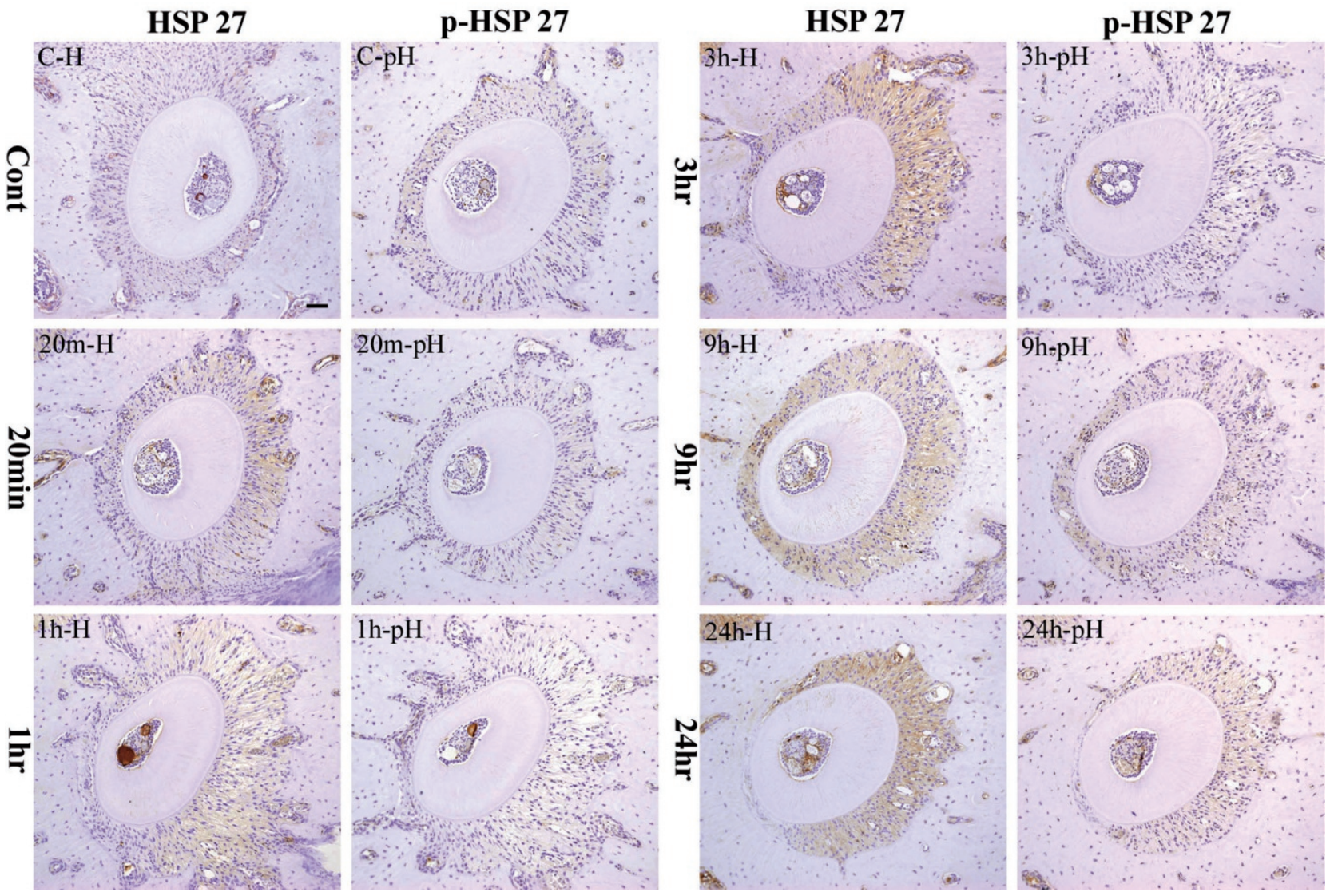

Fig. 5. Immunohistochemical staining profile of HSP27 and p-HSP27 in control and experimental specimens. The inset bar indicates $50 \mu \mathrm{m}$.

fibroblasts increased its eosinophilic staining; had fewer nuclei per unit area. Osteoblasts lining bone surfaces were also fewer (Fig. 4, 3h-P).

In the 9 hours group, eosinophilic staining of the PDL further increased; the number of PDL fibroblasts decreased. Moreover, some obscure spaces partly in the fiber bundles showed hematoxylin staining caused by karyolysis.

In the 24 hours group, the PDL became strongly eosinophilic. Strong nuclear chromatin condensation or pyknosis has been observed in PDL fibroblasts, karyorrhexis is very evident (Fig. 4, 24h-P).

\section{IMMUNOHISTOCHEMICAL EXAMINATION}

\section{Control group}

The PDL of the left maxillary distal buccal root of the mouse first molar weakly expressed HSP27 protein. The expression was mainly detected in the cytoplasm of PDL fibroblasts (Fig. 5, C-H). Expression of pHSP27 was much weaker (Fig. 5, C-pH).

\section{Experimental group}

In the 10 minutes group, HSP expression localized in the PDL fibroblasts in the tension side was slightly stronger compared to the control group. On the other hand, the pressure side was almost negative. However, p-HSP27 was negative in both pressure and tension sides.

In the 20 minutes group, cytoplasmic expression of HSP27 on the PDL fibroblasts seen in the tension and pressure sides was stronger than the 10 minutes group (Fig. 5, 20m-H). There was no change in the p-HSP27 observed when compared to the 10 minutes group (Fig. 5, 20m-pH).

In the 1 hour group, PDL fibroblasts slightly expressed HSP27 stronger compared to the 20 minutes group. No expression was detected in the pressure side (Fig. 5, 1h-H). p-HSP27 was negative in both pressure and tension sides (Fig. 5, 1h-pH).

In the 3 hours group, the PDL fibroblasts and cementoblasts in the tension side strongly expressed HSP27. The expression increased compared to the 1 hour group (Fig. 5, 3h-H). At this point, p-HSP27 expression showed no difference in strength (Fig. 5, 3h-pH).

In the 9 hours group, HSP27 was strongly expressed in the cytoplasm of PDL fibroblasts and cementoblasts as well as in osteoblasts. The expression in the tension side was stronger compared to the 3 hours group (Fig. 5, 9h-H). In this period, p-HSP27 started to become strong in the pressure side (Fig. 5, 9h-pH).

In the 24 hours group, HSP27 was strongly expressed in the tension side by the entire PDL fibroblasts and cementoblasts as well as by osteoblasts. The expression was also stronger compared to the 9 hours group. On the other hand, HSP expression in the pressure side was lost (Fig. 5, 24h-H). Furthermore, the strongest expression of p-HSP27 in the tension side during the whole experiment was detected in this group. In the pressure side, p-HSP27 as well as 
Table 2. Expression Strength of HSP 27and p-HSP27 by IHC

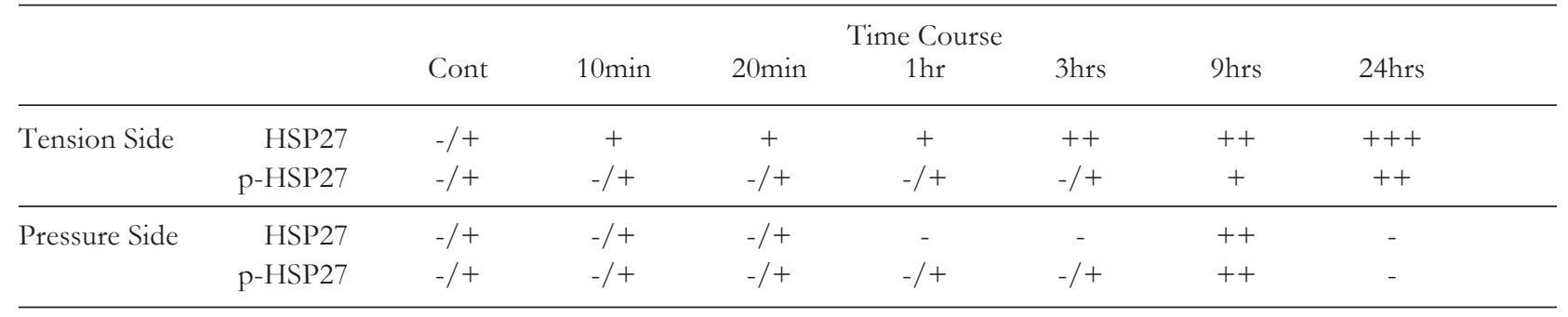

Immunopositivity: -: negative; -/+: weak; +: mild; ++: moderate; +++: strong

HSP27 expressions were not detected (Fig. 5, 24h$\mathrm{ph})$.

Table 2 summarizes the results of the immunohistochemistry.

\section{Discussion}

The mechanism of bone formation and resorption using animal experiments during orthodontic tooth movement has already been clarified. Histopathological studies in the tissue changes due to mechanical stress were observed in alveolar bone and PDL in the pressure and tension sides. In recent years, transcription factors that regulate a variety of morphogenetic pathological response and cellular differentiation have been more widely studied $[1,2]$.

We (Watanabe et al. [3-5]) established the proteins expressed during orthodontic tooth movement by inducing mechanical stress. We investigated the changes in the expressions of Runx 2 and Msx 2 by the PDL fibroblasts. Msx2 expression was usually weak compared to Runx2 which acted as an inhibitor. The expression of Msx2 was facilitated by Runx2 during the regulation of osteoblast differentiation. However, the response of the cells under mechanical stress has not been investigated.

Therefore, we focused on the expression of various HSPs that maintain homeostasis during injury. HSPs are one of the factors recognized that is transiently enhanced by heat shock [9]. It is also called stress protein because it is not only enhanced by heat shock but also by ischemia, other pathological changes such as infections and inflammation, radiation, physical stress such as light, stress from enzymes, heavy-metal ion, arsenic, arsenic acid, methanol, active oxygen and stress from chemical and various amino acid derivatives [10, 11]. In a study by Ritssa [19], HSPs were induced when the chromosomes of Drosophila salivary glands were subjected to high temperatures. Then after, heat shock proteins synthesized in Drosophila was first isolated through SDSPAGE. Some of the major proteins that were also induced by heat shock treatment in non-salivary gland tissues were also collectively considered as HSPs. In addition, puffs from mRNAs of HSPs were transcribed. Later, more advanced researches on HSPs reported that the gene of $E$ coli, yeast and other mammalian cells kept in an environment, subjected to heat stress could preserve the gene [20]. The expression of these HSPs was then proven as a universal phenomenon. HSPs, in ancient times, are proteins acquired in harsh conditions for the survival of cells, induced by non-physiological stimuli, escape from cell death as molecular chaperone [21] and have a functional antiapoptotic property $[13,14]$. Many HSPs are also expressed by cells in response to stress, inhibition and repair of denatured proteins. HSPs are proteins essential for maintaining life functions of various cells during cell differentiation, growth and existence; are widely-distributed intracellular protein equilibrium and are regularly expressed even in non-stressed conditions in in vitro and in vivo experiments [14, 21]. HSP is a dozen to several hundreds KDa polypeptides divided by molecular weight and individually named into high-molecular HSPs (families of HSP110, HSP90, HSP70, HSP40-60) and low-molecular HSPs (HSP20 family). The expressions of these HSPs and functions in different sites have been reported so far.

High-molecular weight HSPs such as HSP70, HSP90, etc temporarily binds to proteins in immature state capable of helping the protein to mature and mediates attachments to polypeptides acting as molecular chaperones [22]. Low-molecular HSPs functioning as molecular chaperones have not yet been disclosed [17].

The investigation of HSP27 belongs to the HSP20 family. Originally, HSP27 was found in actin polymerization [23]. HSP27 are known to be present at high levels in vascular smooth muscle and skeletal muscle cells in non-stimulated state. From this, HSP27 is thought to play a role in maintaining blood pressure and other physiological activities in the vascular system [24].

In addition, Kozawa et al. [25], reported that nonstimulated osteoblast-like MC3T3-E1 cells expressed very low levels of HSP27 (3.5-7.4 ng/mg protein) and has been thought to be involved in controlling the proliferation of osteoblasts. HSP27 is expressed during orthodontic tooth movement particularly in the tension side.

HSP27 functions through a process that is phosphorylated by the action of various kinases ( $p$ HSP27). In osteoblasts, TGF- $\beta 1$ (transforming growth factor beta 1) is phosphorylated by the action of hydrogen peroxide [26] or by p38MAPK (p38MAPkinase) [27]. p38 MAPK was phosphorylated by HSP27 and is thought to be working with HSP27 necessary for the protection of various proteins formed from 140-800 kDa oligomers [28], preventing apoptosis during early stages and cell differentiation [29-31]. In this study, we also examined p-HSP27.

First, with the induction of mechanical stress without specific time, Reitan [32] reported that bone for- 
mation begun 1 to 2 days as a response after simple mechanical stress was applied due to rapid cell-mediated bone remodeling. In addition, temporal expressions of HSP27 and HSP70 polypeptide by PDL cells to mechanical stress have been confirmed [6]. From these things, expressions of HSP27 and p-HSP27 within 24 hours are thought to be involved in cell differentiation. Moreover, Watanabe et al. [3] reported that 20 minutes after mechanical stress applied on mouse molar, the PDL fibroblasts strongly expressed Runx2 and Msx2 in the tension side which increased over time. In this regard, we compared the data of Watanabe et al. [3] from 10 minutes to 24 hours after inserting the rubber dam sheet.

Firstly, histological examination was performed with hematoxylin and eosin stain comparing the control and experimental groups where mechanical stress was induced in the experimental group. Mechanical stress was initially induced at 20 minutes; root relative movement was set up in 3 hours. The PDL space is narrower in the pressure side. Degenerative changes and increased in eosinophilic staining of PDL fibroblasts were observed. PDL fibroblasts nucleus was strongly flattened causing karyolysis. Our findings were similar to those previously described [3$5,8]$.

Immunohistochemical examination was also included in this study. The cytoplasm of the PDL fibroblasts in the distal buccal root of the left maxillary first molar control mice showed weak activity of HSP27 and p-HSP27. Permanent tooth is suffering from mechanical stress due to mastication thousands of times a day. In these circumstances, the supporting PDL maintains its physiological state. In the control group, HSP27 and p-HSP27 are believed to maintain homeostasis of the PDL during physiological function. Similar to reports, HSP27 exists in this non-stress working to maintain homeostasis [14, 21].

When HSP27 and p-HSP27 expression was examined in the experimental group subjected to mechanical stress, PDL fibroblasts expressed HSP27 stronger compared to the control group in the tension side after 10 minutes. HSP27 positive response increased over time; HSP response in 9 hours group on the pressure side increased further by PDL fibroblasts and osteoblasts. Similar to previous reports, HSP is believed to have been involved in repair of cellular damage from injury, damage and various degenerative changes in PDL fibroblasts due to mechanical stress [14, 21]. However, p-HSP27 expression was a bit delayed. This finding suggests that p-HSP27 might function as a molecular chaperone.

Moreover, in the HSP27 expression in 24 hours group; cementoblasts, osteoblasts and the entire PDL fibroblasts in the tension side strongly expressed HSP27. HSP expression in 24 hours group, in the tension side is more intense compared to 9 hours group. It is thought that HSP27 gained its peak between 9 to 24 hours after mechanical stress induction. Also, little variation in the staining of the cytoplasm and nucleus was noted. However, the variation was not clear. p-HSP27 expression in the tension side was the strongest among groups. These reactions are thought to be induced by mechanical stress on the PDL.
In non-stimulated osteoblast-like MC3T3-E1 cells, HSP27 levels were very low (3.5-7.4 ng/mg protein) and that HSP27 was considered to be involved in controlling the proliferation of osteoblasts. Now, in search for HSP27 and p-HSP27 timing expression, the weak expression in the PDL was kept at low levels when not subjected to mechanical stress. This in vivo experiment validates the in vitro data of Kozawa et al. [25]. In addition, after mechanical stress, HSP27 and p-HSP27 were expressed about the same period of time as Runx2, Msx2 and ALP as previously reported by Watanabe et al. [3]. This suggests that HSP27 and p-HSP27 are working as molecular chaperones causing bone formation by activating osteoblasts.

In the pressure side of the 24 hours group, HSP27 and p-HSP27 expressions almost entirely disappeared. Those histological findings are also consistent with the degenerative changes in PDL fibroblasts produced in the pressure side. Moreover, the intense HSP27 expression by the PDL fibroblasts in the tension side more than the 9 hours group was brought by their response to mechanical stress to the cells producing a variety of proteins though long-standing pressure caused the death of other cells. Thus, the force exerted was too much for the PDL and the continuous heat produced due to pressure was not suitable.

In summary, the study suggests that HSP27 has been closely involved in the repair of tissue to maintain homeostasis of the periodontal tissues by the activation of PDL fibroblasts. Also, the data of this study adds to the existing trend in role of Msx2 and Runx2 in the activation of osteoblasts during bone formation suggesting the role of HSP27 as a molecular chaperone during orthodontic treatment induced by mechanical stress.

Acknowledgment: This research was partially supported in part by a Grant-in-Aid for Scientific Research (C) (\#20592419) from the Japan Society for the Promotion of Science.

\section{REFERENCES}

1. Saito Y, Yoshizawa T, Takizawa F, Ikegami M, Ishibashi O, Okuda K, Hara K, Ishibashi K, Obinata M, Kawashima H. A cell line with characteristics of the periodontal ligament fibroblasts is negatively regulated for mineralization and Runx2/Cbfa1/Osf2 activity, part of which can be overcome by bone morphogenetic protein2. J Cell Sci. 2002; 115: 4191-4200.

2. Yoshizawa T, Takizawa F, lizawa F, Ishibashi O, Kawashima H, Matsuda A, Endo N, Kawashima H. Homeobox protein Msx2 acts as a molecular defense mechanism for preventing ossification in ligament fibroblasts. Mol Cell Biol. 2004; 24: 3460-3472.

3. Watanabe T, Nakano K, Muraoka R, Shimizu T, Okafuji N, Kurihara S, Yamada K and Kawakami T. Role of Msx2 as a promoting factor for Runx2 at the periodontal tension sides elicited by mechanical stress. Eur J Med Res. 2008; 13: 425-431.

4. Watanabe T, Okafuji N, Nakano K, Shimizu T, Muraoka R, Kurihara S, Yamada K and Kawakami T. Periodontal tissue reaction to mechanical stress in mice. J Hard Tissue Biol. 2007; 16: 71-74.

5. Watanabe T, Nakano K, Shimizu T, Okafuji N, Kurihara S, Yamada K and Kawakami T. Immunohistochemistry of the Periodontal Ligament Fibroblasts in Orthodontic Tension Sides. J Hard Tissue Biol. 2009: 18(4): 175-180. 
6. Muraoka R, Nakano K, Matsuda H, Tomoda M, Okafuji N, Kurihara S, Yamada K and Kawakami T. Immunohistochemical Observation of Heat Shock Proteins Expression in Mouse Periodontal Tissues due to Orthodontic Mechanical Stress. J Hard Tissue Biol. 2009; 18(4): 193197.

7. Matsuda H, Muraoka R, Tomoda M, Nakano K, Okafuji $\mathrm{N}$, Yamada $\mathrm{K}$ and Kawakami T. Immunohistochemical Observation of BMP in the Mouse Orthodontic Periodontal Tension Sides. J Hard Tissue Biol. 2009; 18(4): 181-184.

8. Kawakami T, Nakano K, Shimizu T, Kimura A, Okafuji $\mathrm{N}$, Tsujigiwa $\mathrm{H}$, Hasegawa $\mathrm{H}$ and Nagatsuka $\mathrm{H}$. Histopathological and immunohistochemical background of orthodontic treatment. Int J Med Biol Front. 2009; 15(7/8): 591-615.

9. Milton, J.S. Heat shock proteins. J Biol Chem. 1990; 265: 12111-12114.

10. Maeda T, Kameda T and Kameda A. Loading of continuously applied compressive force enhances production of heat shock protein 60, 70 and 90 in human periodontal ligament-derived fibroblast-like cells. J Jpn Orthod Soc. 1997; 56: 296-302.

11. Okazaki M, Shimizu Y, Chiba M and Mitani H. Expression of heat shock proteins induced by cyclical stretching stress in human periodontal ligament fibroblasts. Tohoku Univ. dent. J. 2000; 19: 108-115.

12. Craig EA, Weissman JS and Horwich AL. Heat shock proteins and molecular chaperones: mediatora of protein conformation and turnover in the cell. Cell. 1994; 78: 365-372.

13. Arrigo AP and Landry J. Expression and function of the low molecular weight heat shock proteins. In: The Biology of Heat Shock Proteins and Molecular Chaperones. Morimoto RI, Tissières A and Georgopoulos C eds, Cold Spring Harbor Laboratory Press, North America, 1994; pp335-373.

14. Lindquist $\mathrm{S}$ and Craig EA. The Heat-Shock Proteins. Annu Rev Genet. 1988; 22: 631-677.

15. Shigehara S, Matsuzaka K and Inoue T. Morphohistological change and expression of HSP70, osteopontin and osteocalcin mRNAs in rat dental pulp cells with orthodontic tooth movement. Bull Tokyo Dent Coll. 2006; 47(3): 117124

16. Araujo RMS, Oba Y and Moriyama K. Identification of genes related to mechanical stress in human periodontal ligament cells using microarray analysis. J Periodont Res. 2007; 42: 15-22

17. Benndorf R, Hayess K, Ryazantsev S, Wieske M, Behlke J and Lutsch G. Phosphorylation and supermolecular organization of murine small heat shock protein HSP25 abolish its actin polymerization-inhibiting activity. J Biol Chem. 1994; 269: 20780-20784.

18. Waldo CM. Method for the study of tissue response to tooth movement. J Dent Res. 1953; 32: 690-691.

19. Ritossa F. A new puffing pattern induced by temperature shock and DNP in drosophila. Cellular and Molecular. 1962; 18: 571-573

20. Miyagawa Y, Lee JM, Maeda T, Koga K, Kawaguchi Y and Kusakabe T. Differential expression of a Bombyx mori AHA1 homologue during spermatogenesis. Insect Mol Biol. 2005; 14: 245-253.
21. Hratl FU. Molecular chaperone in cellular protein folding. Nature. 1996; 381: 571-579.

22. Gething MJ and Sambrook J. Protein folding in the cell. Nature. 1992; 355: 33-44.

23. Miron T, Vancompernolle K, Vandekerckhove J, Wilchek $\mathrm{M}$ and Geiger B. A $25-\mathrm{kDa}$ inhibitor of actin polymerization is a low molecular mass heat shock protein. J Cell Biol. 1991; 114: 255-261.

24. Inaguma Y, Goto S, Shinohara H, Hasegawa K, Ohshima $\mathrm{K}$ and Kato K. Physiological and pathological changes in levels of the two small stress proteins, HSP27 and alpha B crystallin, in rat hindlimb muscles. J Biochem. 1993; 114: 378-384

25. Kozawa O, Niwa M, Matsuno $H$, Tokuda $H$, Miwa M, Ito $\mathrm{H}$, Kato K and Uematsu T. Sphingosine 1- phosphate induces heat shock protein 27 via p38 mitogen-activated protein kinase activation in osteoblasts. J Bone Miner Res. 1999; 14: 1761-1767.

26. Shibanuma M, Kuroki T and Nose K. Cell-cycle dependent phosphorylation of HSP28 by TGF_1 and H2O2 in normal mouse osteoblastic cells (MC3T3-E1), but not in their RAS-transformants. Biochem Biophys Res Commun. 1992; 187: 1418-1425.

27. Kawamura H, Otsuka T, Matsuno H, Niwa M, Matsui N, Kato K, Uematsu T and Kozawa O. Endothelin-1 stimulates heat shock protein 27 induction in osteoblasts: involvement of p38 MAP kinase. Am J Physiol. 1999; 277: 1046-1054.

28. Kotlyarov A and Gaestel M. Is MK2Åimitogen-activated protein kinase-activated protein kinase $2 \AA$ jthe key for understanding post-transcriptional regulation of gene expression? Biochem Soc Trans. 2002; 30: 959_963.

29. Arrigo AP and Preville X. Role of Hsp27 and related proteins. In: Hand-book of Experimental Pharmacology, DS Latchman ed, Springer, Berlin-New York, 1999; 101-132.

30. Ciocca DR, Oesterreich S, Chamness GC, McGuire WL and Fuqua SA. Biological and clinical implications of heat shock protein 27,000 (Hsp27) a Review. J Natl Cancer Inst. 1993; 85(19): 1558-1570.

31. Mehlen P, Mehlen A, Godet J and Arrigo AP. Hsp27 as a switch between differentiation and apoptosis in murine embryonic stem cells. J Biol Chem. 1997; 272: 3165731665.

32. Reitan K. Tissue behavior during orthodontic tooth movement. Am J Orthodont. 1960; 46: 881-900.

Received: June 4, 2010 / Accepted: July 29, 2010

Address for correspondence:

Rina Muraoka, DDS,

Hard Tissue Pathology Unit,

Matsumoto Dental University

Graduate School of Oral Medicine,

1780 Hirooka-Gobara,

Shiojiri, 399-0781 Japan

Phone and Fax: +81-263-51-2035

E-mail:mura@po.mdu.ac.jp 